\title{
BIOMECHANICALLY BASED MUSCLE MODEL FOR DYNAMIC COMPUTER ANIMATION
}

\author{
Martin Dobšík, ${ }^{1,2}$ Michael Frydrych ${ }^{2}$ \\ ${ }^{1}$ DCGM, FIT BUT, Brno, Czech Republic; ${ }^{2}$ LCE, HUT, Helsinki, Finland \\ dobsik@fit.vutbr.cz, frydrych@Ice.hut.fi
}

\begin{abstract}
Simulation of dynamic motion of soft tissues is one of the most important topic in nowadays computer animation of human body. So far various elastically deformable models were used for this purpose. We present the extension of model proposed in [15] with volume preserving constraint. We provide also evaluation of the constraint accuracy on two simulation experiments. Furthermore we add model of active and passive force generated by musculo-tendon unit using Zajac's bio-mechanical model. We use Finite Difference Method for resolution of continuous partial differential equations of internal elastic forces of the model in space. We integrate resulting ordinary differential equations of motion using various explicit and implicit methods.
\end{abstract}

Keywords: Physically-based modeling, physically-based animation, muscle modeling, deformable model, soft tissue animation, bio-mechanics, simulation

\section{Introduction}

Human body is capable to convey formidable amount of information via it's movement. One can use it to provide a signal to other person, to play small sketch which is telling a story, people can read from our body our emotions. Hence, it is not so surprising that Computer Animation (CA hereafter) of human body was and is widely investigated research field. Although there is good evidence that our body conveys information also on the level of large global movements like rising the hand or turning the head, in many applications it is important to capture the static shape of the body and it's dynamic response to global movement in the most detailed level.

Problem of realistic deformation of soft body tissues was approached from many different angles in the past and present researches. In the beginning there were simple, purely geometric approaches which directly manipulated vertex coordinates depending on the skeleton joint angles [7, 8]. More advanced models, still working on the level of geometry added some higher level features like FFD blocks in [3], Dirichlet FFD [10] or hierarchical B-splines in [5]. 
Another group of approaches adds physical properties to the model by means of physical simulation. In [16] the authors have used mass-spring system to represent skin with it's mechanical behavior. Similar approach for simulating dynamical properties of muscles was used in [11]. Other authors have used Finite Element Method (FEM hereafter). See e.g: [6].

Most of the above mentioned works do not incorporate bio-mechanical properties and limitations into the model. Thus the models, however advanced they are, will always lead to unrealistic deformations under certain conditions. More recent works therefore utilize the knowledge from the field of bio-mechanical engineering, to overcome this problem. As an example let's mention the works [4] and [12].

To implement full model of real bio-mechanical system capturing all its properties is, of course, impossible. Therefore most of the above described research implements very simplified model only. They are usually directed to some specific application like for example the work [11] focuses on real-time simulation, while [4] is "off-line" computational study of a bio-mechanical problem with application to CA.

It is our long term goal: to design and implement dynamic human hand model using anatomical and bio-mechanical knowledge. This paper presents a design of general scalable model for soft objects, and it's extension for realistic computer animation of soft tissues - skeletal muscle in particular. The scalability of the model is meant to allow real-time manipulations in rough resolution, but should also provide high-resolution for final rendering. The work is based on the works of $[2,15,4]$.

\section{Continuous formulation of the Model}

The model we propose is derived from the one published by [15]. Authors of the original work provide details for modeling curves, surfaces and solids. We describe the solid model only, referring the reader to the previously mentioned work for details.

We can describe motion of all points $\boldsymbol{r}(\boldsymbol{a}, t)$ of deformable body, on infinitesimal level, using Lagrange's equations of motion:

$$
\mu \frac{\partial^{2} \boldsymbol{r}}{\partial t^{2}}+\frac{\delta \varepsilon(\boldsymbol{r})}{\delta \boldsymbol{r}}+\frac{\delta \nu(\boldsymbol{r}, \boldsymbol{v})}{\delta \boldsymbol{v}}+\frac{\delta \kappa(\boldsymbol{r})}{\delta \boldsymbol{r}}=\boldsymbol{F}(\boldsymbol{r}, t)
$$

where: $\boldsymbol{r}(\boldsymbol{a}, t)=\left[r_{x}, r_{y}, r_{z}\right]$ is position of particle with local parametric coordinates $\boldsymbol{a}=\left[a_{1}, a_{2}, a_{3}\right]$ at time $t ; \mu(\boldsymbol{a})$ is mass density of the material at $\boldsymbol{a}$; $\varepsilon(\boldsymbol{r})$ is a functional measuring potential energy of deformation; $\frac{\delta}{\delta \boldsymbol{r}}(f)$ is variational derivative of a functional $f ; \frac{\delta \varepsilon(\boldsymbol{r})}{\delta \boldsymbol{r}}$ describes the elastic forces restoring deformed body to it's undeformed shape; $\boldsymbol{F}(\boldsymbol{r}, t)$ are external forces. Dissipation $\frac{\delta \nu(\boldsymbol{r}, \boldsymbol{v})}{\delta \boldsymbol{v}}$ and volume preservation forces $\frac{\delta \kappa(\boldsymbol{r})}{\delta \boldsymbol{r}}$ will be described in next paragraphs. 
Elastic Forces and Dissipation. The approximation of variational derivative of internal energy functional $\varepsilon(\boldsymbol{r})$ (see [15]) and dissipative functional $\nu(\boldsymbol{r})$ (see [2]) can be expressed:

$$
\begin{aligned}
& \frac{\delta \varepsilon(\boldsymbol{r})}{\delta \boldsymbol{r}}+\frac{\delta \nu(\boldsymbol{r}, \boldsymbol{v})}{\delta \boldsymbol{v}} \approx \boldsymbol{e}(\boldsymbol{r}, \boldsymbol{v})=-\sum_{i, j=1}^{3} \frac{\partial}{\partial a_{i}}\left(\left(\alpha_{i j}+\gamma_{i j}\right) \frac{\partial \boldsymbol{r}}{\partial a_{i}}\right) \\
& \text { where: } \quad \alpha_{i j}(\boldsymbol{a}, \boldsymbol{r})=\eta_{i j}(\boldsymbol{a})\left(G_{i j}-G_{i j}^{0}\right), \quad \boldsymbol{E}=\boldsymbol{G}-\boldsymbol{G}^{0}, \\
& G_{i j}(\boldsymbol{r}(\boldsymbol{a}))=\frac{\partial \boldsymbol{r}}{\partial a_{i}} \cdot \frac{\partial \boldsymbol{r}}{\partial a_{j}}, \\
& \gamma_{i j}(\boldsymbol{a}, \boldsymbol{r}, \boldsymbol{v})=\varphi_{i j}(\boldsymbol{a}) \dot{E}_{i j}, \\
& \dot{E}_{i j}(\boldsymbol{r}(\boldsymbol{a}), \boldsymbol{v}(\boldsymbol{a}))=\frac{1}{2} \dot{G}_{i j}=\frac{\partial \boldsymbol{r}}{\partial a_{i}} \cdot \frac{\partial \boldsymbol{v}}{\partial a_{j}}+\frac{\partial \boldsymbol{r}}{\partial a_{j}} \cdot \frac{\partial \boldsymbol{v}}{\partial a_{i}},
\end{aligned}
$$

tensor $\eta_{i j}(\boldsymbol{a})$ defines the "stiffness" of material at point $\boldsymbol{a}$ in the direction $(i, j)$, tensor $\gamma_{i j}(\boldsymbol{a})$ describes the dissipation density the same way. $\boldsymbol{G}$ is current metric tensor at point $\boldsymbol{r}(\boldsymbol{a})$ and $\boldsymbol{G}^{0}$ is metric tensor when the model is in its rest shape. $\dot{\boldsymbol{E}}=\partial \boldsymbol{E} / \partial t$ represents "speed" of deformation. Please see [15, 2] for further details.

Incompressibility. We are developing model suitable for animation of soft tissues of human body. However, most of the soft tissues are quasi-incompressible (see e.g. [9]). The model described so far does not resist to volume change. We want the model to preserve it's volume during deformation so we want to maintain the condition: $V-V^{0}=0$. To achieve this we can use functional:

$$
\kappa(\boldsymbol{r})=\int_{\Omega}\left\|d V-d V^{0}\right\|_{\psi}^{2} d a_{1} d a_{2} d a_{3}, \text { where } d V=\left|\frac{\partial \boldsymbol{r}}{\partial a_{1}} \cdot \frac{\partial \boldsymbol{r}}{\partial a_{2}} \times \frac{\partial \boldsymbol{r}}{\partial a_{3}}\right|
$$

is volume of infinitesimal element. $d V^{0}$ is volume of the same element in rest shape. The same way as in case of elastic energy and dissipation, we can find minimum of integral (3) from calculus of variation. The resulting term:

$$
\begin{aligned}
\frac{\delta \kappa(\boldsymbol{r})}{\delta \boldsymbol{r}} \approx \boldsymbol{k}(\boldsymbol{r})= & 2 \psi\left(d V-d V^{0}\right) \cdot\left[-\frac{\partial}{\partial a_{1}}\left(\frac{\partial \boldsymbol{r}}{\partial a_{2}} \times \frac{\partial \boldsymbol{r}}{\partial a_{3}}\right)\right. \\
& \left.+\frac{\partial}{\partial a_{2}}\left(\frac{\partial \boldsymbol{r}}{\partial a_{1}} \times \frac{\partial \boldsymbol{r}}{\partial a_{3}}\right)-\frac{\partial}{\partial a_{3}}\left(\frac{\partial \boldsymbol{r}}{\partial a_{1}} \times \frac{\partial \boldsymbol{r}}{\partial a_{2}}\right)\right]
\end{aligned}
$$

will force model from equation (1) to preserve constant volume. Term (4) introduces extra internal forces to the model attempting to push the model into such deformation which preserves the volume. As a consequence of this formulation the volume of the model during deformation will not be exactly constant, but will return to it's original value in equilibrium. However, we will show in section 5 that the deviation of volume during animation is negligible.

\section{Bio-mechanical properties}

Human body is composed of hundreds of muscles of different kind. Our aim is to model muscles which serve as a motors for movement of body. We 
have chosen to model the linear muscle with pennation since it is very common skeletal muscle and is very well described by number of different biomechanical models. We chose to simulate this complex mechanical device, using model of F. E. Zajac, which have already been succesfully used in several computer animation projects (e.g $[4,12])$.

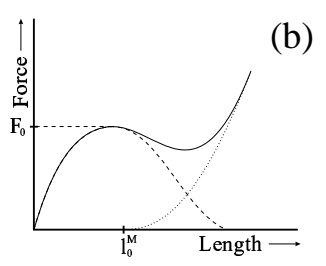

(b)

(a)

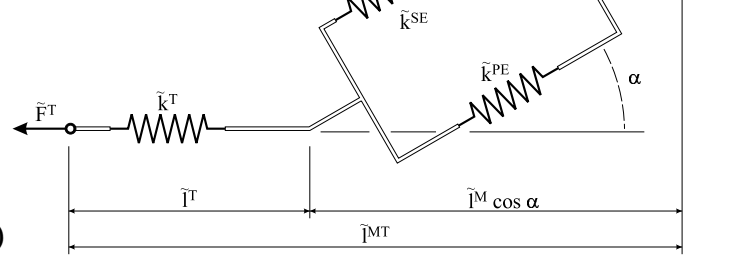

(d)

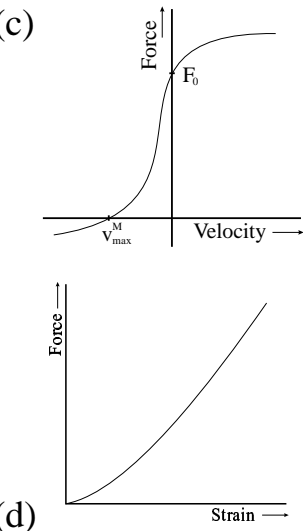

$\mathrm{a}(\mathrm{t})$... activation signal

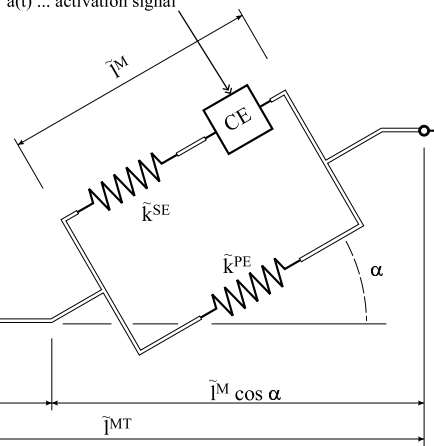

(c)
Figure 1. Zajac's model: (a) the scheme of mechanical arrangement; (b) dashed line-active muscle force $(\mathrm{CE})$, dotted line - passive muscle force $\left(k^{P E}\right)$, solid line - active + passive; (c) velocity dependence of active force; (d) passive tendon force $\left(k^{T}\right)$ length dependence.

Zajac's Dimensionless Musculo-tendon Model is designed for complete musculo-tendon unit. Figure 1a) shows the model schematically. Characteristics of interest (length, force, speed) are all described without dimensions. We use normalized versions of quantities. As an example, actual tendon length $l^{T}$ has normalized equivalent $\tilde{l}^{T}=\frac{l^{T}}{l_{S}^{T}}$. Zajac measured characteristics of isolated muscle fiber and tendon. They can be easily scaled to simulate complete muscle. Parameters describing the model are: $\alpha \ldots$ pennation angle; $f_{0}^{M}$ ... maximum isometric force of active muscle; $l_{0}^{M} \ldots$ optimal muscle length at which $f_{0}^{M}$ is developed; $l_{s}^{T} \ldots$ tendon rest length; $v_{\max }^{M} \ldots$ maximum shortening velocity of muscle fibers. Figures $1 b$ ) to $1 d$ ) describe their meaning. We refer the reader to $[9,4]$ for further details about the model.

We tackle the inclusion of these bio-mechanical properties into our model similar way as the authors of [4]. See next section for details.

\section{Discretization in Space}

Now we have continuous formulation of dynamic deformable volume preserving model with approximate physically based dissipation and we have description of bio-mechanical characteristics. The model is described by equa- 
tion of motion (1) and terms (2), (4) and by Zajac's model. We use Finite Difference Method (FDM hereafter) to solve these equations numerically.

FDM simply discretizes continuous space domain into discrete set of nodal points. In case of our three-dimensional parametric model we create $M \times$ $N \times O$ nodal points on parametric space $0 \leq a_{1}, a_{2}, a_{3} \leq 1$ with internode spacing $h_{1}=1 /(M-1), h_{2}=1 /(N-1), h_{3}=1 /(O-1)$. Thus we approximate continuous vector function $\boldsymbol{r}\left(a_{1}, a_{2}, a_{3}\right)$ by the grid function $\boldsymbol{r}[m, n, o]=\boldsymbol{r}\left(m h_{1}, n h_{2}, o h_{3}\right)$.

To evaluate (2) and (4) we need discrete approximation of first and second partial derivatives of nodal variables - difference operators (DO hereafter). Large number of DO are used in theory and practice of FDM. However, as previous work [13] and also our investigation shows, the simplest one are most appropriate due to the nature of equations we are solving. We use the same DO as are proposed in original work [15] and justified in [13]: first order forward and backward DO and central and cross DO; with natural (free) boundary conditions. Derivation of resulting approximations using DO is straightforward.

Adding Musculo-Tendon Properties. The deformable model described so far serves as the model for passive material properties of muscle. We add the nonlinear active and passive muscle force generators between the mesh nodal points obtained from FDM to simulate muscle contraction. Another nonlinear force generators for tendons are added to connect the muscle to bone. Situation is described on figure 2 .

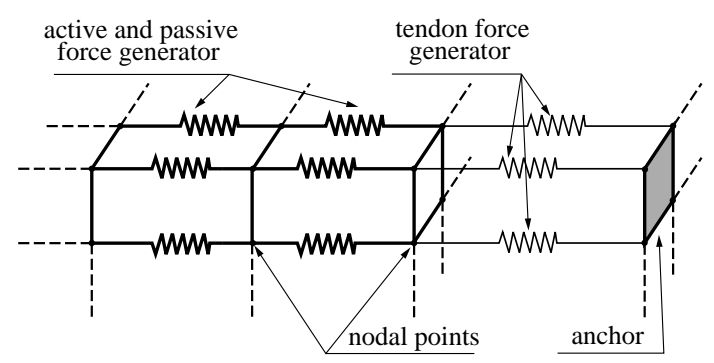

Figure 2. Model of active muscle fi ber from fi gure 1b) and 1c) is added in one axial direction in between every successive nodal points. Muscle is attached to fi xed plane at both ends with tendon model from fi gure 1d).

\section{Resolution of Equations of Motion in Time}

After discretization in space using FDM we can arrange all nodal points into one vector $\overline{\boldsymbol{r}}$ (For example 2D model discretized into $2 \times 3=6$ nodal points would be written as: $\left.\overline{\boldsymbol{r}}=[\boldsymbol{r}[1,1] \boldsymbol{r}[1,2] \boldsymbol{r}[1,3] \boldsymbol{r}[2,1] \boldsymbol{r}[2,2] \boldsymbol{r}[2,3]]^{T}\right)$. Let us use $\boldsymbol{r}_{i}$ to refer to $i$ th element of vector $\overline{\boldsymbol{r}}$. Using this notation we can write our 
eqn of motion (1) for each nodal point $i$ independently:

$$
m_{i} \ddot{\boldsymbol{r}}_{i}+\boldsymbol{e}_{i}(\overline{\boldsymbol{r}}, \dot{\overline{\boldsymbol{r}}})+\boldsymbol{k}_{i}(\overline{\boldsymbol{r}})+b i o_{i}(\overline{\boldsymbol{r}}, \dot{\overline{\boldsymbol{r}}})=\boldsymbol{F}_{i}(\overline{\boldsymbol{r}}, t)
$$

where $\dot{\boldsymbol{r}}=\frac{\partial \boldsymbol{r}}{\partial t}, \ddot{\boldsymbol{r}}=\frac{\partial^{2} \boldsymbol{r}}{\partial t^{2}}$, bio $(\overline{\boldsymbol{r}}, \dot{\overline{\boldsymbol{r}}})$ represents bio-mechanical forces from figure 1 and $m$ is the mass of nodal point $i$. Rearranging and substitution gives two $1^{\text {st }}$ order ordinary differential equations (ODE hereafter) of two variables:

$$
\begin{array}{ll}
\dot{\boldsymbol{r}}_{i}=\mathrm{g}_{\mathbf{i}}(\mathbf{t}, \overline{\boldsymbol{v}}) & =\boldsymbol{v}_{i} \\
\dot{\boldsymbol{v}}_{i}=\mathbf{f}_{\mathbf{i}}(\mathbf{t}, \overline{\boldsymbol{r}}, \overline{\boldsymbol{v}}) & =\frac{1}{m_{i}}\left(\boldsymbol{F}_{i}(\overline{\boldsymbol{r}}, t)-\boldsymbol{e}_{i}(\overline{\boldsymbol{r}}, \overline{\boldsymbol{v}})-\boldsymbol{k}_{i}(\overline{\boldsymbol{r}})-b i o_{i}(\overline{\boldsymbol{r}}, \overline{\boldsymbol{v}})\right)
\end{array}
$$

We are currently running evaluation of integration methods for this model. So far we have experimented with explicit methods: Euler, $2^{\text {nd }}$ and $4^{\text {th }}$ order Runge-Kutta, Cash-Carp Runge-Kutta of $5^{t h}$ order with adaptive step size control, Adams-Bashforth multi-step, Adams-Moulton predictor-corrector multistep [14] and implicit Euler method [1]. Qualitative and quantitative evaluation of different integration methods will be the topic of another study.

\section{Results and Examples}

Our implementation of simulation environment is made in $\mathrm{C}++$ in multiplatform environment using OpenGL for visualization. It allows us to simulate 3D parametric model of general passive material, active muscle model and also 2D parametric models as described in original work [15]. It implements also a variety of numerical integration methods as described above. The simulation is carried in real-time allowing interactive manipulation of the model.

Figure 3 shows the brick of passive material whose topmost plane is fixed in space (hanging down the ceiling). Pictures (a) and (b) have smaller isotropic parameter $\eta$ then pictures (c) and (d).

Active Musculo-Tendon Model with applied gravitational forces is shown on figure 4. Tendons on both sides of muscle are attached to fixed plane. Simulation of equations of motion for muscle is much more complicated due to nonlinearities introduced via Zajac's bio-mechanical model. See figure captions for details.

\section{Discussion and Future Work}

We presented general scalable model with it's application to skeletal muscle animation. It appears to have good properties with respect to stability and accuracy so that it can serve as a good starting point for hand model implementation. We have experimented with variety of numerical resolution methods and material parameters for the model and have shown 2 subtle example model implementations. However, the nonlinearities introduced to model of muscle cause the eqn of motion to be more difficult to solve. Our aim is, therefore, to 


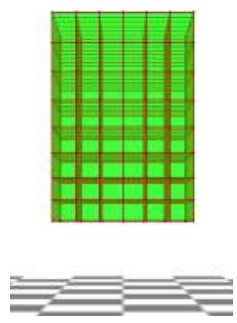

(a)

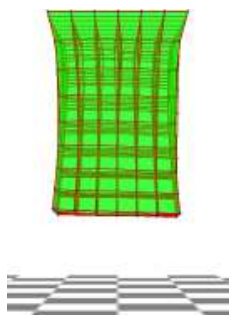

(b)

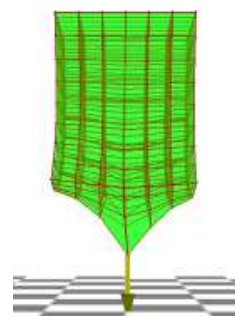

(c)

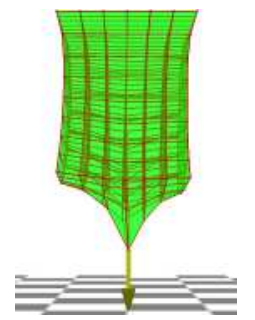

(d)

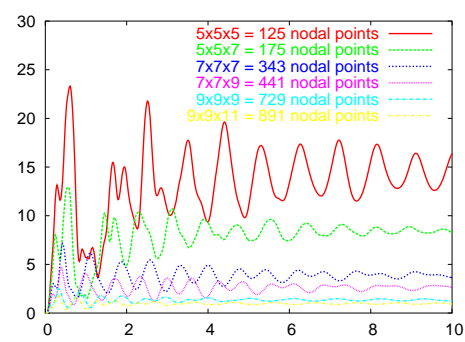

(e)

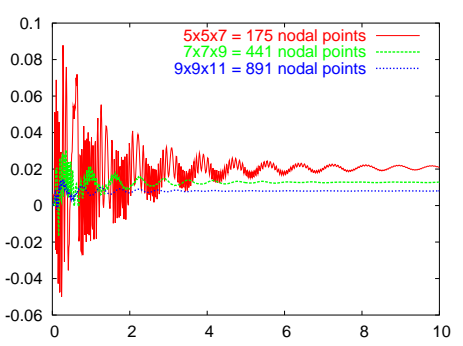

(f)

Figure 3. Passive model under influence of external forces is shown on top row; (a),(b) model without and with volume preservation forces; (c),(d) another external force added. (e) deviation of total volume ([\%] change in time) vanishes as the accuracy of discretization (number of nodal points) increases. (f) deviation of sum of $\mathrm{dV}$ from eqn (3) in [\%] with increasing resolution. Values on (e) and (f) are from animation whose fi nal frame is displayed on fi gure (d).

(a)

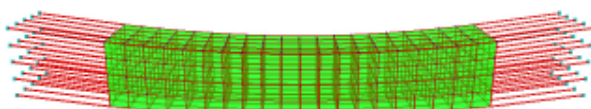

(b)
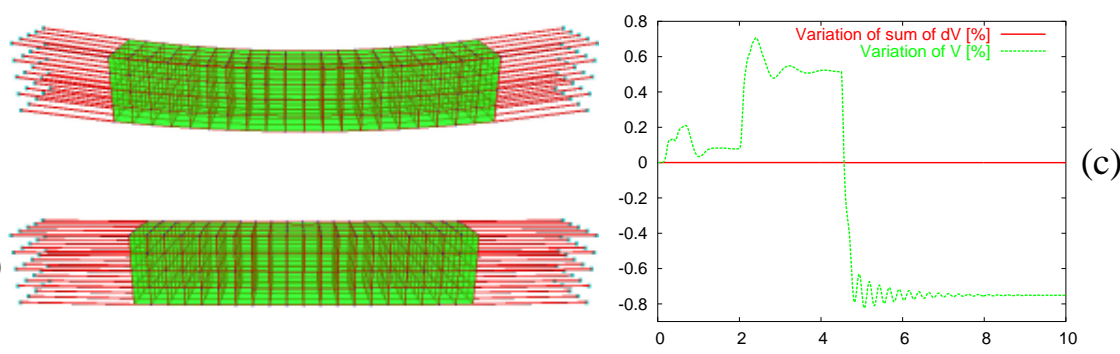

Figure 4. Active musculo-tendon model is shown in its relaxed state on (a) and when activated via model of neural excitation on (b). Graph (c) shows the deviation of the sum of $d V$ and of total volume from the rest state, evolving in time during simulation. Note that right attachment plane was moved suddenly $8 \%$ of muscle length to the right, after 2 seconds of simulation. After 4.5 seconds was muscle activated. Discretization is $21 \times 5 \times 5=525$ nodal points.

investigate thoroughly various numerical simulation methods and design and implement new one which would allow much faster computation of more complex systems with several muscles in real-time.

We will continue in our research to complete the simplified hand model with bones and skin to allow driving the hand using neural excitation control. For 
this we need to incorporate also collision detection and response. We will also validate the muscle model via performing similar bio-mechanical experiments as Zajac did with real muscle and as in [4] the authors did using FEM.

Acknowledgments: This work was partly supported by CIMO Fellowship program for young researchers (www.cimo.fi), EU Research Training Network Multi-modal Human-Computer Interaction (HPRN-CT-2000-00111) and by the Czech Ministry of Education (Research intention no. CEZ: J22/98: 262200012 - Research in information and control systems).

\section{References}

[1] D. Baraff and A. Witkin. Large Steps in Cloth Simulation. In SIGGRAPH'98, pages 43-54, 1998.

[2] M. Carignan, Y. Yang, N. M.-Thalmann, and D. Thalmann. Dressing animated synthetic actors with complex deformable clothes. In CGI'92, pages 99-104. ACM Press, 1992.

[3] E. Chadwick, John, R. Haumann, David, and E. Parent, Richard. Layered construction for deformable animated characters. In SIGGRAPH'89, pages 243-252, 1989.

[4] T. D. Chen and D. Zeltzer. Pump It Up: Computer Animation of a Biomechanically Based Model of Muscle Using the Finite Element Method. In SIGGRAPH'92, pages 89-98, 1992.

[5] D. R. Forsey. A Surface Model for Skeleton-Based Character Animation. In Proceedings of the Eurographics Workshop on Animation and Simulation, pages 55-73, 1991.

[6] J.-P. Gourret, N. Magnenant-Thalmann, and D. Thalmann. Simulation of Object and Human Skin Deformations in a Grasping Task. In SIGGRAPH '89, pages 21-30, 1989.

[7] R. Laperriere, N. Magnenant-Thalmann, and D. Thalmann. Joint-Dependent Local Deformations for Hand Animation and Object Grasping. In Graphics Interface'88, 1988.

[8] N. Magnenant-Thalmann and D. Thalmann. The Direction of Synthetic Actors in the Film Rendez-vous à Montréal. IEEE CG\&A, pages 41-51, 1988.

[9] W. Maurel, Y. Wu, N. Magnenat-Thalmann, and D. Thalmann. Biomechanical Models for Soft Tissue Simulation. Springer-Verlag Berlin/Heidelberg, 1998.

[10] L. Moccozet. Hand Modeling and Animation for Virtual Humans. PhD thesis, University of Geneva, 1996.

[11] L. P. Nedel and D. Thalmann. Real Time Muscle Deformations Using Mass-Spring Systems. In Computer Graphics Interface, 1998.

[12] V. Ng-Thow-Hing. Anatomically-based models for physical and geometric reconstruction of humans and other animals. PhD thesis, University of Toronto, 2001.

[13] P. Palmer, A. Mir, and M. Gonzáles. Stability and Complexity Study of Animated Elastically Deformable Objects. In Hans-Helmut Nagel and Francisco J. Perales López, editors, AMDO'2000, pages 58-71, 2000.

[14] W. H. Press, B. P. Flannery, S. A. Teukolsky, and W. T. Vetterling. Numerical Recipes: The Art of Scientific Computing. Cambridge University Press, 2nd edition, 1992.

[15] D. Terzopoulos and K. W. Fleischer. Deformable models. The Visual Computer, 4(6):306-331, December 1988.

[16] R. Turner and D. Thalmann. The Elastic Surface Layer Model for Animated Character Construction. In Computer Graphics International, 1993. 